\title{
Neurosurgical considerations in supratentorial low-grade gliomas: experience with 175 patients
}

\author{
Dimitrios C. Nikas, M.D., Lorenzo Bello, M.D., Amir A. Zamani, M.D., and Peter McL. Black, \\ M.D., Ph.D. \\ Divisions of Neurosurgery and Neuroradiology, Brigham and Women's Hospital, Children's Hospital; \\ and Departments of Surgery and Radiology, Harvard Medical School, Boston, Massachusetts
}

The authors reviewed 175 low-grade hemispheric gliomas surgically treated by one surgeon (P.B.) between 1987 and 1996: 74 astrocytomas (42\%), 35 oligodendrogliomas (20\%), 52 mixed gliomas (30\%), 12 gangliogliomas (7\%), and two ependymomas (1\%). Patient age ranged from 7.5 to 81.9 years (mean 39.2 years); 84 patients (48\%) were males and 91 (52\%) females. Postsurgical follow-up review ranged from 0.1 to 225.2 months (mean 36.2 months, median 24.9 months). Either $\mathrm{T}_{2}$-weighted or contrast-enhanced $\mathrm{T}_{1}$-weighted magnetic resonance (MR) images were used to evaluate the percentage of resection achieved and volume of residual disease postoperatively. The majority of patients (55\%) had seizures as the presenting symptom, and $45 \%$ experienced preoperative symptoms for more than 12 months. Tumor enhancement was present in $21 \%$ of cases. In $66 \%$ of surgical procedures at least one of the following technical adjuncts was used: monitored local anesthesia, real-time MR imaging, stereotactic guidance with computerized tomography, three dimensional reconstruction, cortical mapping with cortical stimulation, somatosensory or visual evoked potential recording, corticography, or intraoperative ultrasound. Intraoperative MR imaging was used for $40(22.9 \%)$ of the craniotomies and nine $(5.14 \%)$ biopsies. There were no surgery-related deaths. Complications appeared in $6 \%$ of the patients. Progression to a higher-grade tumor occurred in $9.2 \%$ of patients within the 3 -year follow-up period.

Key Words * low-grade glioma * local anesthesia * real-time magnetic resonance imaging * prognostic factors

Supratentorial low-grade gliomas represent one of the most challenging tumors in neurooncology. They are a diverse and heterogeneous group of tumors, the biological activity of which can be quite variable. Despite several studies, their natural history and management remain unclear, in part because of confusing pathological classifications and terminology. Confusion also arises because of conflicting data from numerous retrospective studies in which different parameters in populations of patients are examined, inhomogeneous and usually not sufficiently large to have statistical significance.

Of the 14,500 brain tumors newly diagnosed each year in the U.S., approximately one-half are primary 
brain tumors of glial origin. Of these, close to $25 \%$ are low-grade astrocytomas. This results in approximately 1800 new cases of low-grade astrocytoma per year; in other words, one out of eight patients suffering from a brain tumor has a low-grade astrocytoma. In the combined results of studies obtained in 1980 and 1985, astrocytoma, pilocytic astrocytoma, and oligodendroglioma represented $26.6 \%, 1.2 \%$, and $2.1 \%$, respectively, of all newly diagnosed cases of primary brain tumor.[48]

In our institution surgery plays a critical role in the management of a low-grade glioma; [7] precise diagnosis, confirmation of histology and grade, and appropriate surgical and adjuvant therapy all play a significant part in the ultimate fate and quality of survival of these patients. Results of surgical intervention have traditionally been measured in terms of patient survival, but recently time to recurrence or malignant transformation have become of critical importance. To define the influence of surgery alone is not always easy, because subsequent radiation therapy may influence the results. $[12,14,43,46,85]$ Rather, our knowledge is based on retrospective studies which used different parameters and prognostic characteristics that encompass both the pre-computerized tomography (CT) and CT era, use variable criteria to measure the degree of surgical resection, and are not easily comparable.

\section{CLINICAL MATERIAL AND METHODS}

\section{Patient Population}

Between 1987 and 1996, 175 patients harboring low-grade gliomas underwent 258 operations performed by the senior author (P.B.). These represent $38 \%$ of the gliomas surgically treated by the same surgeon during that period. Of these 175 patients, 91 were female and 84 male. The average age at presentation was 39.2 years. The majority of patients $(55 \%)$ had seizures as the presenting symptom, and $45 \%$ had preoperative symptoms for more than 12 months. Forty-six percent of these patients underwent surgery only, whereas the rest underwent radiation therapy, stereotactic radiosurgery, or chemotherapy as adjuvant treatment. We followed these patients for a mean period of 36.2 months, (median 24.9 months) and $9.2 \%$ of them had tumor that progressed to a higher grade.

\section{Pathological Classification}

Grading systems for astrocytomas differ in the relative importance attributed to individual histological criteria, but most systems measure rate, by using cytological atypia, mitotic activity, microvascular proliferation, and necrosis. As a result, various classifications have been proposed at different times in an attempt to relate them to the diverse structural aspects adopted by normal and reactive astrocytes and to predict the clinical expression from the microscopic appearance of the neoplasm. Kernohan's classification and the newer St. Anne/Mayo classification are systems of grading from 1 to 4 in ascending order of malignancy.[18] Histological grading systems for oligodendroglioma are less predictive of outcome than those of astrocytomas, and there is disagreement as to which criteria are useful.[19] The term anaplastic oligodendroglioma is restricted to a relatively pure type of rapidly growing, highly cellular, and poorly differentiated tumor with increased mitotic activity and the presence of necrosis. The tumors in our study were considered low grade either by St. Anne/Mayo or World Health Organization classification systems. The majority of the tumors were astrocytomas, (42\%), oligodendrogliomas, (20\%), mixed gliomas (30\%), and gangliogliomas (7\%).

\section{Neuroradiological Evaluation}

In all patients preoperative workup tests included magnetic resonance (MR) imaging and in most cases CT scanning. Tumor enhancement was present in $21 \%$ of cases. Postoperative assessment was performed 
by MR and/or CT imaging 3 to 6 months postsurgery.

\section{RESULTS}

The distribution of tumors, as well as age and gender, among the 175 patients with low-grade gliomas who underwent surgery at our institution are shown in Table 1. The patient age distribution showed a peak incidence between 35 and 40 years. The median age was 37 years (range 7.5-81.9 years). There was no significant difference in the gender of patients with these tumors.

\begin{tabular}{|c|c|c|c|c|c|c|}
\hline \multicolumn{7}{|c|}{$\begin{array}{c}\text { TABLE } 1 \\
\text { SUMMMRY OF TUMOR TVPE \& PATIENT CHARACTER STICS IN } 175 \text { SURGICALLY TREATED } \\
\text { LOW-GRADE GLIOMSS }\end{array}$} \\
\hline & \multicolumn{5}{|c|}{ Tumor Type } & \multirow[b]{2}{*}{ Total } \\
\hline & $\begin{array}{l}\text { Astro- } \\
\text { cytoma }\end{array}$ & $\begin{array}{l}\text { Oligoden- } \\
\text { droglioma }\end{array}$ & $\begin{array}{l}\text { Mixed } \\
\text { Glioma }\end{array}$ & $\begin{array}{l}\text { Gangio- } \\
\text { glioma }\end{array}$ & $\begin{array}{c}\text { Eper } \\
\text { dymona }\end{array}$ & \\
\hline no. of patients (\%) & $74(42)$ & $35(20)$ & $52(30)$ & $12(7)$ & $2(1.1)$ & $175(100)$ \\
\hline $\begin{array}{l}f \\
m\end{array}$ & $\begin{array}{l}32(18.3) \\
42(24)\end{array}$ & $\begin{array}{l}19(10.9) \\
16(9.1)\end{array}$ & $\begin{array}{l}31(17.7) \\
21(12)\end{array}$ & $\begin{array}{l}7 \text { (4) } \\
5(2.9)\end{array}$ & $\begin{array}{l}2(1.1) \\
0\end{array}$ & $\begin{array}{l}91(52) \\
84(48)\end{array}$ \\
\hline $\begin{array}{l}\text { age (yrs) } \\
\text { average } \\
\text { median } \\
\text { range }\end{array}$ & $\begin{array}{l}39 \\
36.4 \\
18.3-81.9\end{array}$ & $\begin{array}{c}38.5 \\
36.3 \\
7.5-72.9\end{array}$ & $\begin{array}{l}40.9 \\
36.5 \\
15.3-73.4\end{array}$ & $\begin{array}{l}35.6 \\
30.8 \\
21.9-57.6\end{array}$ & $\begin{array}{c}45.5 \\
45.5 \\
24.9-66\end{array}$ & $\begin{array}{l}39.2 \\
37 \\
7.5-81.9\end{array}$ \\
\hline
\end{tabular}

Of the different treatment modalities, $46 \%$ of patients underwent surgery only, whereas the remaining patients underwent radiation treatment, stereotactic radiosurgery, or chemotherapy as additional treatment. The majority of the patients underwent a surgical procedure, ranging from biopsy sampling to gross-total tumor removal followed by postoperative radiation therapy. The different treatment modalities are given in Tables 2 and 3. The mean follow-up period was 36.2 months (Table 4), during which 16 tumors $(9.2 \%)$ progressed to a higher grade (Table 5).

\begin{tabular}{|c|c|c|c|c|c|c|}
\hline \multicolumn{7}{|c|}{$\begin{array}{c}\text { TABLE } 2 \\
\text { SURGKAL TECHN QUE USED IN } 175 \text { PATIENTS UNDERGONG FIRST OPERATIONA }\end{array}$} \\
\hline \multirow[b]{2}{*}{ Tumor Type } & \multicolumn{6}{|c|}{ No. of Patients (\%) } \\
\hline & $\begin{array}{l}\text { Crani- } \\
\text { otomy }\end{array}$ & $\begin{array}{l}\text { Stere o- } \\
\text { tactic } \\
\text { Biopsy }\end{array}$ & $\begin{array}{l}\text { Stere o- } \\
\text { tactic } \\
\text { Crani- } \\
\text { otomy }\end{array}$ & $\begin{array}{l}\text { MRT } \\
\text { Crani- } \\
\text { otomy }\end{array}$ & $\begin{array}{c}\text { MRT } \\
\text { Biopsy }\end{array}$ & $\begin{array}{c}\text { Open } \\
\text { Brain } \\
\text { Biopsy }\end{array}$ \\
\hline $\begin{array}{l}\text { astrocytoma } \\
\text { oligodendroglioma } \\
\text { mixed Gioma } \\
\text { ganglioglioma } \\
\text { epend ymoma. } \\
\text { total }\end{array}$ & $\begin{array}{l}26(14.9) \\
14(8) \\
23(13) \\
9(5.1) \\
2(1.1) \\
73(41.2)\end{array}$ & $\begin{array}{l}20(11.4) \\
9(5.1) \\
7(4) \\
0 \\
0 \\
36(20.6)\end{array}$ & $\begin{array}{c}7(4) \\
1(0.6) \\
6(3.4) \\
2(1.1) \\
0 \\
16(9.1)\end{array}$ & $\begin{array}{l}18(10.3) \\
8(4.6) \\
12(6.85) \\
1(0.6) \\
0 \\
40(22.9)\end{array}$ & $\begin{array}{l}3(1.7) \\
3(1.7) \\
3(1.7) \\
0 \\
0 \\
9(5.1)\end{array}$ & $\begin{array}{l}0 \\
0 \\
1(0.7) \\
0 \\
0 \\
1(0.6)\end{array}$ \\
\hline
\end{tabular}




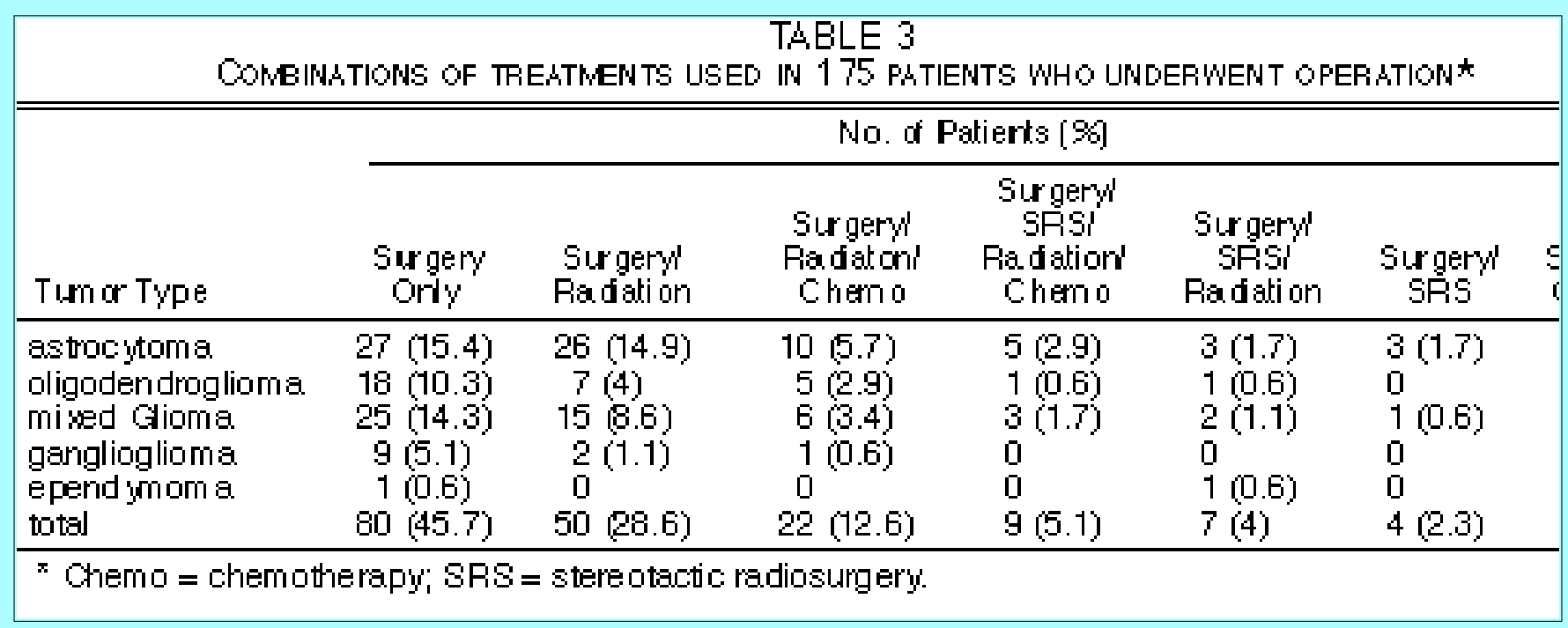



\section{DISCUSSION}

Based on our results, clinical experience, and review of the literature, our conclusions can be summarized as follows.[9]

1) Low-grade gliomas are tumors in young adults that may transform into more malignant types. The median age of our patients was 37 years, and this is almost certainly indicative of specific features of the pathophysiology of their low-grade gliomas. The reason for this higher incidence in young patients is not clear. It is important to recognize, however, that these low-grade tumors transform to higher-grade tumors. Patients with low-grade tumors usually die from malignant recurrence. In our series $9.2 \%$ of the tumors recurred in a higher grade during the follow-up period (Fig. 1). 

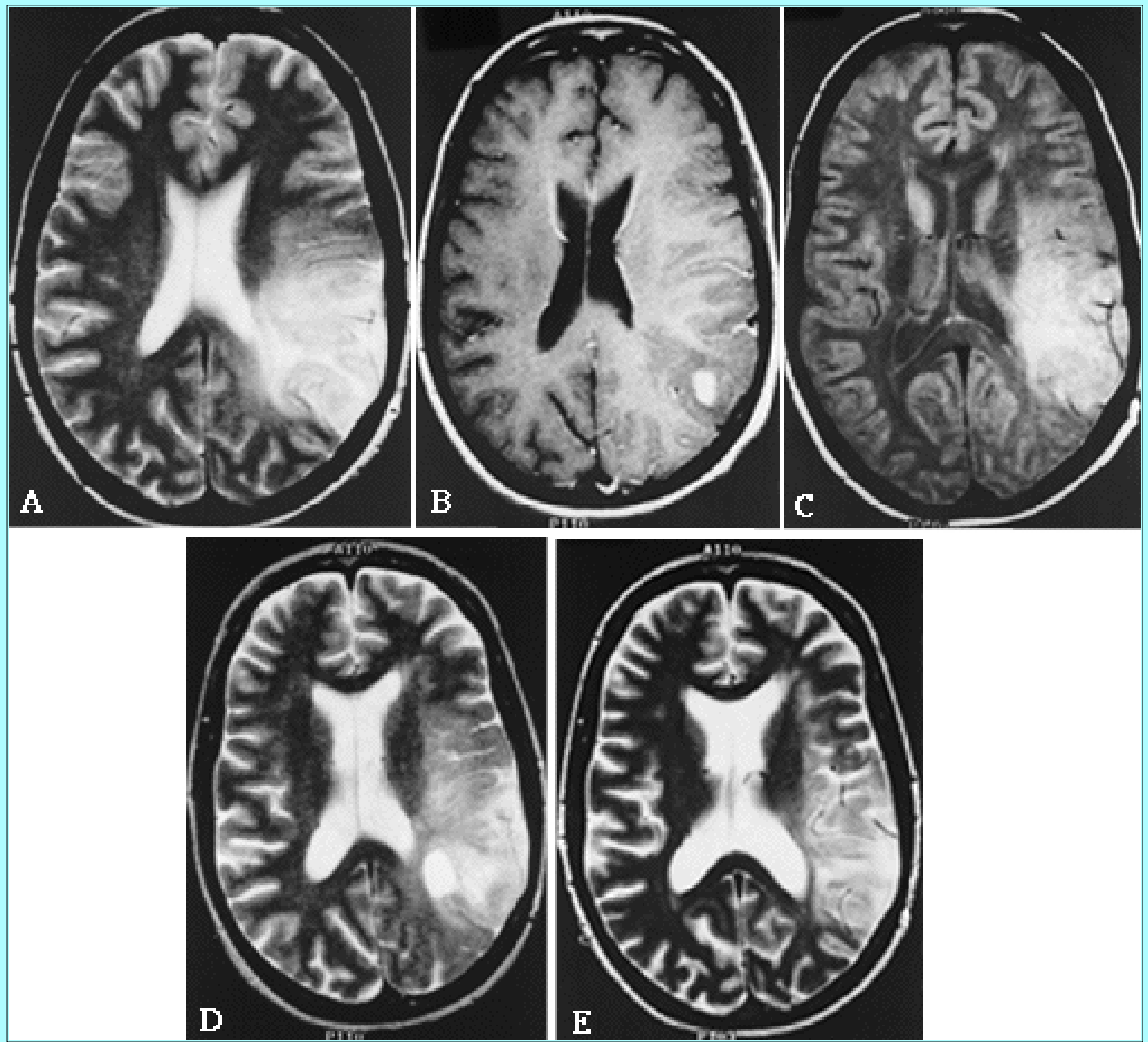

Fig. 1. Dedifferentiation of low- to high-grade tumor tissue demonstrated using MR tomography (MRT)-guided laser ablation in a female patient. A: Nonenhancing infiltrating tumor of the left temporoparietal lobe, with minimal mass effect. Biopsy sampling followed by radiation therapy was performed. B: After 4 years, the patient presented with increased seizure frequency and an enhancing lesion in the left parietal area revealed by MR imaging. C: A biopsy sample of the lesion was obtained in the MRT suite and found to be an anaplastic astrocytoma. From the various treatment options, MRT-guided laser ablation of the focal lesion with subsequent chemotherapy was elected. Imaging following the procedure demonstrated no significant amount of edema and no intracranial hemorrhage. D: Three months later, decreased enhancement with residual enhancement remaining along the medial aspect of the lesion and decreased overall mass effect were demonstrated. E: One year later, the surgical cavity has resolved; however, there is slight persistent nodular enhancement in this region and $\mathrm{T}_{2}$ signal abnormality within the cortex and white matter.

The percentage of recurring astrocytomas that showed anaplastic areas in a second biopsy specimen or at autopsy varies in different series from 13 to $85 \%$.[21,26,40,49,50,56,61,66,67] The histological 
modification at recurrence can be attributed either to occurrence of malignant transformation or to the expression of an aggressive component in the original tumor that was missed on the tissue specimen submitted for pathological review.[77] The benefit of surgery seems to result from a significant decrease in the risk of recurrence in patients who underwent aggressive surgical treatment, compared to those receiving anything less than gross-total resection.[61,62] Berger, et al.,[2] have reported on the association between extent of resection and recurrence, using a univariate analysis, in a series of 53 patients with heterogeneous tumor types (19 astrocytoma, 18 oligodendroglioma, and 16 mixed oligoastrocytoma). Analysis of results in this study suggested that both tumor size at presentation and after resection may influence the natural history of low-grade gliomas. Patients with low-grade gliomas and a tumor volume greater than $10 \mathrm{~cm}^{3}$ benefit from radical surgery at the time of radiographic diagnosis, in terms of influencing the incidence of recurrence, time to tumor progression, and malignant transformation. Berger and colleagues suggested that the biology of large $\left(>10 \mathrm{~cm}^{3}\right)$ low-grade gliomas is quite different from smaller tumors. All recurrences of big tumors demonstrated malignant transformation, which was not the case with tumors in the 10 to $30 \mathrm{~cm}^{3}$ range. In patients with tumors less than $10 \mathrm{~cm}^{3}$ there were no recurrences. In a series conducted by Laws, et al.,[41] approximately one-half of the low-grade tumors tended to remain true to grade whereas $49 \%$ progressed. Eighty percent of the patients with Grades 1 and 2 tumors in the series of Soffietti, et al.,[77] had Grade 3 or 4 tumors at the time of clinical recurrence or death. Recurrent tumor growth of Grade 1 astrocytomas (9\%) was comparatively infrequent in the series by Firsching, et al.,[23] but was a common phenomenon (62\%) in Grade 2 tumors. Vertosick, et al.,[84] observed in their series that the only factor that could predict in which patient dedifferentiation would occur was diagnosis at a younger age compared with those dedifferentiating later in life (mean age 33 vs. 43 years, respectively). Piepmeier, et al.,[62] have stated that tumors causing chronic epilepsy are much less likely to evolve into anaplastic lesions and more typically behave as benign astrocytomas, whereas other histologically similar low-grade astrocytomas maintain a potential for more malignant evolution.[1] In another study, patients with low-grade gliomas that evolved to anaplastic tumors did not significantly differ in the 5-year survival rate (22\%) from matched de novo patients (28\%).[20]

2) Low-grade tumors have a very complex histopathology that may reflect differing potential for growth. The classification of tumor subtypes is quite controversial and seems to vary from center to center. The distinction between mixed gliomas, pure oligodendrogliomas, and fibrillary astrocytomas is sometimes very difficult to make.[36,37,45] The differing tissue types and classifications may represent differing cells of origin for these tumors.

Difference in age, localization, and choice of criteria in grading can partly explain the discrepancies among the different studies. Features such as cell density, nuclear pleomorphism, mitoses, vessel frequency and size, hyperplasia, perivascular infiltrates, microcysts, microcalcifications, and the use of proliferation markers[81] have been studied, and attempts have been made to connect these factors with survival. Different institutions use different classification systems usually not specifically described in the published series, and this makes comparisons difficult and produces wide variations in prognoses in the literature.[15] For example, Grade 2 lesions according to the Kernohan four-tiered system would be anaplastic astrocytomas in the three-tiered system proposed by Burger.[13] When the St. Anne/Mayo Clinic grading system[18] was used by Janny, et al.,[31] tumor grade was the principal variable on which survival of patients with ordinary hemispheric astrocytomas depended. In this study the reclassification of patients from the Kernohan to St. Anne/Mayo Clinic grading systems resulted in the emergence of anaplastic tumors initially classified as low grade. The St. Anne/Mayo Clinic system was found by the 
authors to distinguish among the four conventional grades with simplicity, objectivity and reproducibility. Using the Kernohan system, Fazekas[22] reported a better survival rate in patients with Grade 1 astrocytomas as compared to those with Grade 2 astrocytomas. The same conclusion was reached by Philippon, et al.,[63] and Leibel, et al.,[43] who found a marked survival advantage in patients with Grade 1 over Grade 2 tumors, although those with Grade 2 might have included higher grades and Grade 1 may have included the pilocytic variety, which is found to behave extremely favorably.[25,47]

On the contrary, Laws, et al.,[42] and North, et al.,[58] found that histological subtype had no influence on survival rates. Soffietti, et al.,[77] found vessel size to be the only histological factor of prognostic significance. Tumor capillaries with a diameter more than $12 \mu \mathrm{m}$ and without endothelial hyperplasia were significantly associated with shorter duration of survival $(\mathrm{p}<0.01)$. The presence of few mitoses did not influence median survival. Nicolato, et al.,[56] performed an analysis of 11 histological parameters, and the only significant histological prognostic factor discerned was presence of microcalcifications $(\mathrm{p}=0.03)$, whereas in the Cohadon, et al.,[15] series nuclear pleomorphism, neovascularization, and necrosis were associated with survival.

Correlation of different histological subtypes with survival led to the definition of distinct pathological entities with different biological behavior.[25] Gangliogliomas[38] and dysembryoplastic neuroepithelial tumors exhibit benign characteristics, whereas the gemistocytic variety behaves as an anaplastic rather than low-grade glioma. Gangliogliomas, as long as they do not present histological evidence of anaplasia, have a relatively favorable prognosis (median survival time was 90.3 months).[30] Dysembryoplastic neuroepithelial tumors, usually located frontally and temporally, present with seizures in young adults, and no recurrences were reported in the series by Daumas-Duport, et al.[17] On the contrary, gemistocytic astrocytoma has been shown to occur in an older age group (38-48 years), and the survival reported (34 months) is shorter than that for ordinary astrocytomas.[67]

3) Low-grade gliomas may occupy eloquent areas of cortex, but there is increasing evidence that their surgical resection is the most important factor in their prognosis, and aggressive surgery appears to increase survival. It is of considerable interest that low-grade gliomas are the only type of glioma for which survival in patients has increased over the last decade, as evaluated by the Central Brain Tumor Registry of the United States. This may be a result of both improved surgical treatment[69] and earlier detection. Given the propensity of these tumors to transform, aggressive surgical resection, with acceptable morbidity and mortality rates, should be carried out as early as possible to reduce the pool of neoplastic astrocytes and to decrease the statistical chance that dedifferentiation will occur. Because they are not diffusely infiltrative in all cases and they may transform into higher grades, we believe that every attempt at complete resection should be made.

The overall 5-year survival rate for these patients is about 50\%,[67] a fact that proves the potentially malignant nature of this tumor and urges for more accurate determination of their natural history if we want to actively prolong the survival rates in these patients. This has not been successful up until now because of several difficulties, in both diagnostic and therapeutic approaches: the tumors present with a varied histological pattern at multiple sites of origin with an unpredictable rate of growth and heterogeneity of clinical behavior; there is no reliable method of predicting the difference in the prognosis; different optimum treatment modalities have been tried; and prolonged follow-up periods are needed to evaluate the influence of the various therapeutic schemes. 
Several clinical, neuroradiological, therapeutic, and histological prognostic factors have been described for ordinary astrocytomas, the most common type of low-grade glioma. In an attempt to combine information regarding 1356 patients treated between 1956 and 1985, one study came up with a 5-year survival rate of 48\%.[68] In initial reports on a series of patients surgically treated by Cushing, the median survival rate was between 2 and 3 years, [83] and in another series it was 23 months for patients who underwent operation before 1960.[28] Survival gradually improved, and a large Mayo Clinic series demonstrated that survival was better in patients whose tumors were diagnosed after 1950 than those diagnosed before.[39,40] In this study, patients who were 20 years of age or older had a 5-year survival rate of $30 \%$. In more recent reports performed in the CT era, it has been found that optimum therapy can yield a 5-year survival rate of 40\%,[52] and median survival times of 7.15 years[61] and 8.2 years[84] have been reported, whereas Piepmeier, et al.,[61] with a minimum follow-up period of 5 years, observed an astonishingly high median survival rate of 12 years and a 5-year survival rate of greater than $90 \%$.

Generally the median survival rate is primarily a function of the time period in which tumors were diagnosed in the patients. In the absence of high-resolution scanning with contrast enhancement before the availability of CT scanners, some high-grade lesions could have been misdiagnosed as low grade.[84] Earlier diagnosis in the CT era permitted early and more effective management. The question remains whether the improvement in survival was a result of better treatment or just earlier diagnosis.[62] Comparison between these two groups, therefore, is not easy because patients present with different clinical pictures. Patients in the pre-CT era were diagnosed after the low-grade tumor had grown enough to cause mass effects, and symptoms like headache, papilledema, and focal neurological signs were the common presenting symptoms, $[28,40,77,83]$ whereas the CT era patient population with astrocytomas usually present with seizures. In the clinical series presented by Vertosick, et al.,[84] 80\% of patients presented with a new seizure disorders with no findings on physical examination. Patients in this category, who harbor a low-grade glioma with no mass effect, would likely not have been considered candidates for surgical treatment or even biopsy before CT technology. The authors attributed the long survival rates they observed (median survival 8.2 years) mainly to the early diagnosis of astrocytomas in patients who exhibited only seizures. Advances in therapeutic modalities also contributed to the observed increase in survival over the last 50 years. Advances in preoperative and postoperative care, pharmaceutical agents, better anesthesia, and radiotherapy could have played a crucial role in the longer survival times even though the absence of a prospective, randomized clinical trial makes the evaluation of these parameters impossible. The use of different classification systems and the fact that not all studies respect in detail the precise pathological criteria, which also varied from time to time, makes the extraction of definite conclusions concerning the improvement of survival rates more difficult.

The benefit of radical surgical resection on the survival of patients has been the subject of numerous retrospective studies and reviews that evaluate the role of surgical removal or simple biopsy followed, or not, by other treatment modalities.[22,32,44,70,74-76,79,80,82,86,87] In these reports the population of the patients studied is heterogeneous and subject to different combinations of adjuvant treatments. The evaluation of the extent of resection is usually based on the judgment of the surgeon, which may not be reliable. There is a potential selection bias. Patients from whom biopsy samples are taken or whose tumors are partially resected often have highly infiltrating lesions, and significant debulking of the lesion is often not possible as the tumor encroaches on functionally vital structures. Those who undergo radical resection usually have tumors that do not infiltrate deep structures, and thus they may constitute a group with more favorable prognosis. In an analysis of the available literature, Berger and Rostomily[6] concluded that approximately half of the studies that analyze exclusive sets of CT-era patients using 
multivariate analysis find greater extent of resection to be associated with longer survival. Although most attention has been directed at this issue with regard to high-grade astrocytomas[55] and there is no solid proof derived from reviewing the literature, there does appear to be a consensus regarding the extent of tumor resection and subsequent prognosis, and there are indications that aggressive surgery, when possible, results in better patient outcome.

In a recent report Piepmeier, et al.,[61] found gross-total tumor resection the most important variable affecting survival; the benefit for these patients seemed to result from a significant decrease in the risk of recurrence. In another study it was found that $80 \%$ of patients with complete removal were alive at 5 years postsurgery compared with $50 \%$ with incomplete resection and $45 \%$ with biopsy only.[63] These figures are very similar to the results of Laws, et al.,[42] and Soffietti, et al:[77] the 5-year survival rate was $61 \%$ with total tumor removal, $44 \%$ for incomplete resection, and $31 \%$ for biopsy in the former study, whereas in the latter series the 5-year survival rate was $51 \%$ with total tumor removal and $23 \%$ with subtotal resection. When appropriate stratification variables such as age, tumor volume, radiation technique, and tumor location are used, the possible benefit of cytoreductive surgery may be less convincing. North, et al.,[58] found that patients who underwent radical or subtotal resection fared significantly better when compared with patients from whom only biopsy samples were taken. However, individuals selected for radical resection tended to have tumors that were easily accessible, appeared less invasive on imaging studies, and were smaller in size.

Surgery is also suggested by Janny, et al.,[31] as the most effective treatment, provided that only Grades 1 and 2 tumors are considered along with a gross-total or subtotal resection. The 5- and 10-year survival rates of patients satisfying these two criteria were $87.5 \%$ and $68.2 \%$, respectively, compared with $57 \%$ and $31.2 \%$, respectively, for patients with Grades 1 and 2 tumors and partial or no surgery. Similarly, the median time of tumor recurrence was significantly longer in the first subgroup. Thus, surgery, although it does not cure those patients, may, because of the slow growing tumor, give them a relatively long survival time provided that the resection has been large enough. Reichenthal, et al.,[65] found that patients who underwent radical subtotal resection had a 5-year survival rate that was nearly twice that of patients in whom only a biopsy sample was taken.

On the other hand, there are studies that question the value of radical surgery. Miralbell, et al.,[51] did not find an association between extent of resection and survival time. In univariate analysis, residual tumor volume less than $23 \mathrm{ml}$ was associated with a better outcome, a conclusion that proved insignificant in multivariate analysis. Vertosick, et al.,[84] reported one of the longest yet-to-be published median survivals of 8.2 years in a study in which the majority $(80 \%)$ of their 25 patients were treated by obtaining a biopsy sample only; they asserted that aggressive therapy of low-grade tumors may need to be reevaluated. There was no incidence of mortality or major morbidity, and the results were in accord with earlier series of Scanlon and Taylor.[70] However, the authors stated that median survival might have been longer if radical surgery had been performed early; thus, in the absence of controlled data, they could not argue that radical surgery has no role. Shaw and colleagues[72,73] examined the survival distribution for different degrees of surgical resection and found that the survival rates were similar for gross-total, radical subtotal, subtotal removal, and biopsy alone, with 5- and 10-year survival rates of approximately $52 \%$ and $23 \%$, respectively. In another study Recht, et al.,[64] compared the survival rates of a group of 20 surgically treated patients, with a histologically proven low-grade hemispheric astrocytoma, to a group of 26 patients who were supposed to have a low-grade tumor based on neuroradiological characteristics and their clinical presentation: 15 of 26 patients of the second group developed progressive disease based on the worsening of their clinical status, increasing tumor size, or 
the development of contrast enhancement on a CT or MR images. The two groups did not differ substantially in observed survival rates.

Extent of resection has been correlated not only with survival but also with time to tumor recurrence. Steiger, et al.,[78] reported that the frequency of tumor progression is lower after total resection. They also reported that a well-demarcated tumor boundary is associated with a better outcome compared to a diffuse margin. Easier radical resection for circumscribed tumors is not the only reason for the better survival of the well-delineated versus diffusely infiltrating lesions. A well-circumscribed tumor may have an inherently low proliferative potential.[81] Tumor infiltration is obviously the consequence of the migrating activity of cells. There may also be a link between histological tumor appearance and migrating activity; for example, a pilocytic architecture can be understood as a consequence of absent cellular migration. This concept would explain the favorable prognosis of pilocytic astrocytomas by the association of low proliferative and low migrational potential. The same issue has been raised by Medbery, et al.,[50] who followed patients who had undergone complete surgical resection without receiving postoperative radiation therapy; they found that all were believed to be cured of their tumors 6.5 to 21.5 years after resection. The tumors in these patients belonged to the subset of well-circumscribed lesions which generally can be curatively resected. On the other hand, in patients whose tumors are not well encapsulated or defined and therefore cannot be completely excised without undue morbidity, there was no survival advantage to heroic efforts at debulking compared to a more limited surgical procedure.

4) Surgery under local anesthesia is an extremely important element in tumor management, and this modality poses substantial challenges. We have performed craniotomies in more than 250 patients after induction of local anesthesia and monitored conscious sedation to secure maximum tumor excision with minimum postoperative deficits in tumors involving eloquent cortex.[16,57] Low-grade gliomas comprise the majority of tumors surgically treated after induction of local anesthesia. The infiltrating nature of these tumors as well as the fact that they occur in a relatively young population, with a better prognosis after complete excision, makes this histologically varied type of tumor a very good candidate for resection. In all the cases, the main indication was presence of the tumor in close proximity to eloquent areas in either hemisphere. Low-grade gliomas near speech or motor cortices are the most typical candidates, but stereotactic craniotomy for deeper lesions is also performed after induction of local anesthesia if lesions are near an internal capsule or visual pathways. The bulk of the tumors was at the frontal and temporal region. Most patients were referred because their tumor was considered inoperable by conventional craniotomy after induction of general anesthesia. The size of the tumor did not determine our decision to proceed with induction of local anesthesia. Most of the tumors were of intermediate size (between 3-6 cm), but many cases were larger.

Because lesions such as low-grade gliomas can distort the normal topography of the cerebral cortex and vascular landmarks for defining cortical zones can also exhibit a great degree of spatial variability among patients, we have used cortical mapping before tumor resection to define the limits of a safe resection, especially for lesions around the motor strip and in areas essential for language and recent memory function.[3-5,10,24,27,29,60] A number of different anesthetic regimens were used, but the majority of cases were managed using a combination of infusions and small boluses of midazolam, fentanyl, and/or sufentanil or propofol, midazolam, fentanyl, and/or sufentanil. A small number of patients were treated with various combinations of these medications in conjunction with esmolol, droperidol, ketamine, and/or brevital. The majority of operations were performed with patients in the supine position after induction of anesthesia. With this technique, complete or substantial resection was achieved in 
three-fourths of the patients with a low rate of new neurological deficit.

Most of the operative procedures were completed without any complication. In a few, the problems were mild and readily controllable. Generally, the patients tolerated the procedure well. Many of them commented that they were relieved to help in monitoring their own neurological condition during tumor removal. When one considers the invasiveness of the procedure and the potential helplessness of the patient, the absence of any significant psychological sequelae is noteworthy. A patient's perception of the event, with its life-prolonging purpose carried out voluntarily by a trusted surgeon, probably best explains the absence of significantly adverse sequelae. Patient agitation, when present, was generally controlled with personal contact, additional medication, or lessening of medication. Complaints of pain were treated by additional local infiltration and opiate. If patient drowsiness interfered with monitoring or mapping, we weaned the patient from medication and a small delay ensued. The most painful part of the procedure was the incision and reflection of the scalp, muscle, and periosteum. In the event that brief, generally focal seizures appeared, intravenous medication was always able to control them.

We reviewed 157 consecutive cases performed after induction of local anesthesia.[16] Complete resection, confirmed by postoperative imaging, was achieved in $23 \%$ of cases and substantial but incomplete resection (> 80\%) was achieved in 57\%. The principal reasons for incomplete resection were pathological involvement of cortex proven to be eloquent by cortical mapping or the development of an intraoperative deficit. In $20 \%$ of the cases, partial resection or simple biopsy was all that could be achieved. In our series the overall incidence of new major neurological deficit was very low (4\%). The majority of early postoperative neurological deficits resolved soon. The development of an intraoperative neurological deficit was a good predictor of early postoperative deficit, but its absence did not preclude such a deficit. Intraoperative neurological monitoring has proven to be a very useful adjunct to cortical mapping in the detection of these intraoperative neurological deficits and in the prevention of permanent major deficits. Most early postoperative deficits did not lead to permanent postoperative ones. The presence of postoperative complications not associated with the performance of craniotomy after induction of local anesthesia did not differ in frequency from the general anesthesia cases. The percentage of surgical complications requiring reoperation was very small. Operations using local anesthesia induction were not associated with any lengthening of the operating time or the length of stay in the hospital, both major components of the cost of treatment. The mean operating room time usage was 4.7 hours, with a range of 1.7 to 8 hours. The mean operating room time for craniotomy for brain tumor at Brigham and Women's Hospital (BWH) in the years between 1992 to 1995 has remained constant at 5.7 hours, although this figure includes some lengthy skull base procedures. The mean length of stay for this series of patients was 5.4 days, with a range of 2 to 19 days. This may be compared with a mean length of stay for all patients undergoing craniotomy for brain tumors at BWH, which was 8.4 days in 1992, dropping gradually to 5.9 days in 1995. Furthermore, with our technique no major additional use of hospital resources beyond those required for other brain tumor craniotomies was needed. In terms of specialized personnel, no additional staff was needed, except for a neurophysiological technician who was required to set up and administer the cortical stimulator.

We found language localization the most difficult task to perform due to the subtlety of this function. The investigation of language localization with electrical stimulation mapping during neurosurgical operations after induction of local anesthesia provides a different perspective on the generally accepted model. Substantial individual variability in the exact location of language function, sometimes correlated with the patient's sex and verbal intelligence, has been described, and this has major clinical implications for cortical resections of the dominant hemisphere, because language cannot be reliably localized on 
anatomical criteria alone. A maximum resection with minimum risk of postoperative aphasia requires individual localization of language with a mapping technique.[59]

5) Better imaging techniques and image-guided surgery, most recently including intraoperative MR imaging, have made a major difference in identification of the tumor and completeness of resection. Using a variety of imaging techniques, the surgeon can see the extent and location of the tumor well. Three-dimensional (3-D) reconstruction techniques in our surgical planning laboratory (Figs. 2 and 3) have allowed us to very precisely identify the tumor, its relation to motor cortex, language areas, blood vessels, and brainstem, and its overall configuration. $[8,35,54]$

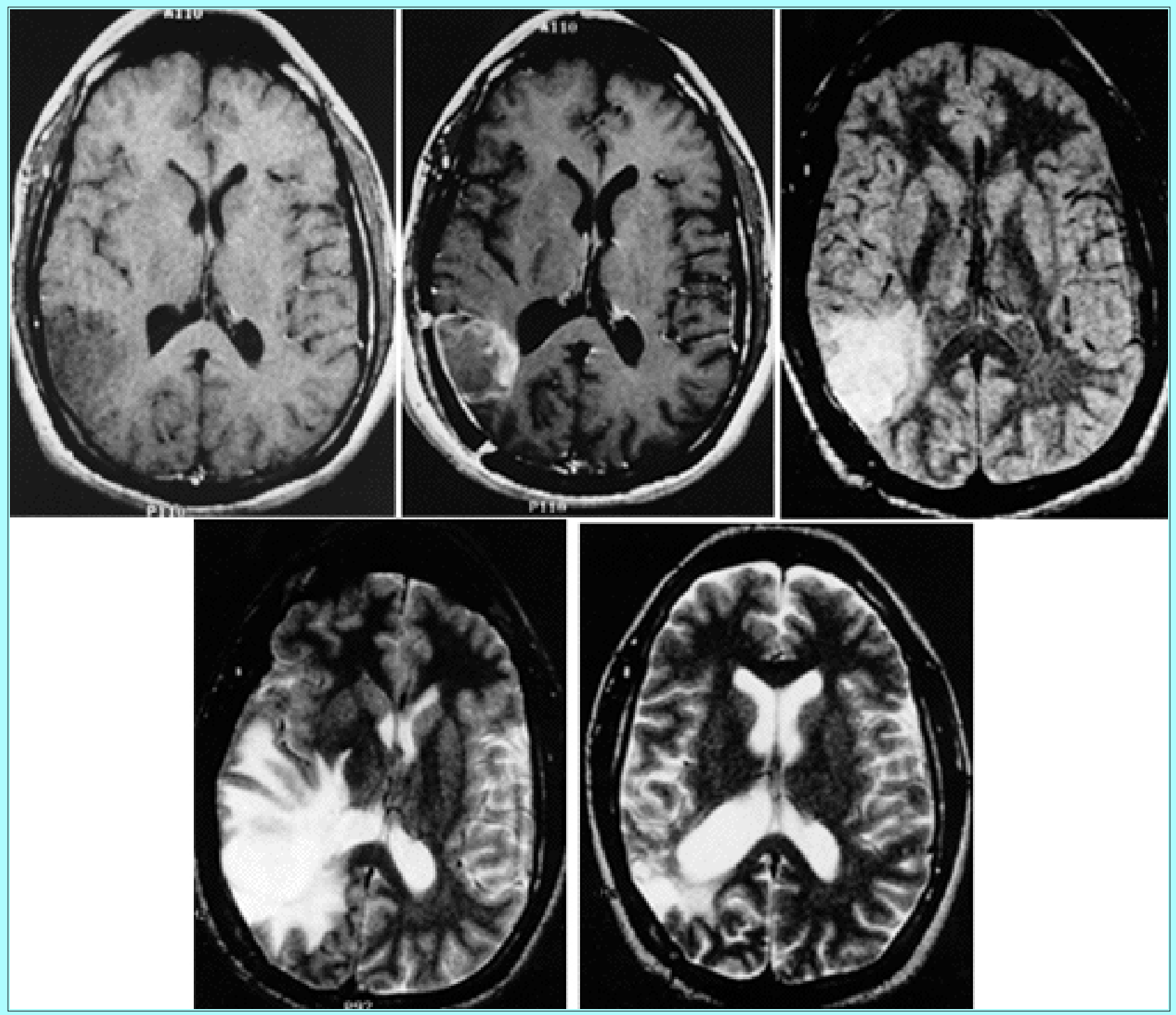

Fig. 2. Aggressive treatment of a Grade 2 astrocytoma by using 3-D reconstruction and motor and sensory mapping. A: Right temporoparietal nonenhancing lesion. B: Substantial resection with residual $T_{1}$ high signal intensity filling the cavity and slight postoperative nodular enhancement of the cavity. C: Recurrence of tumor, surrounded by edema, 6 months following treatment. D: Progression of enhancing mass lesion with significantly worse edema and mass effect. The heterogeneous enhancement pattern of the lesion suggests radiation changes. E: Postoperative changes with encephalomalacia and some ex vacuo dilatation of the right lateral ventricle. 

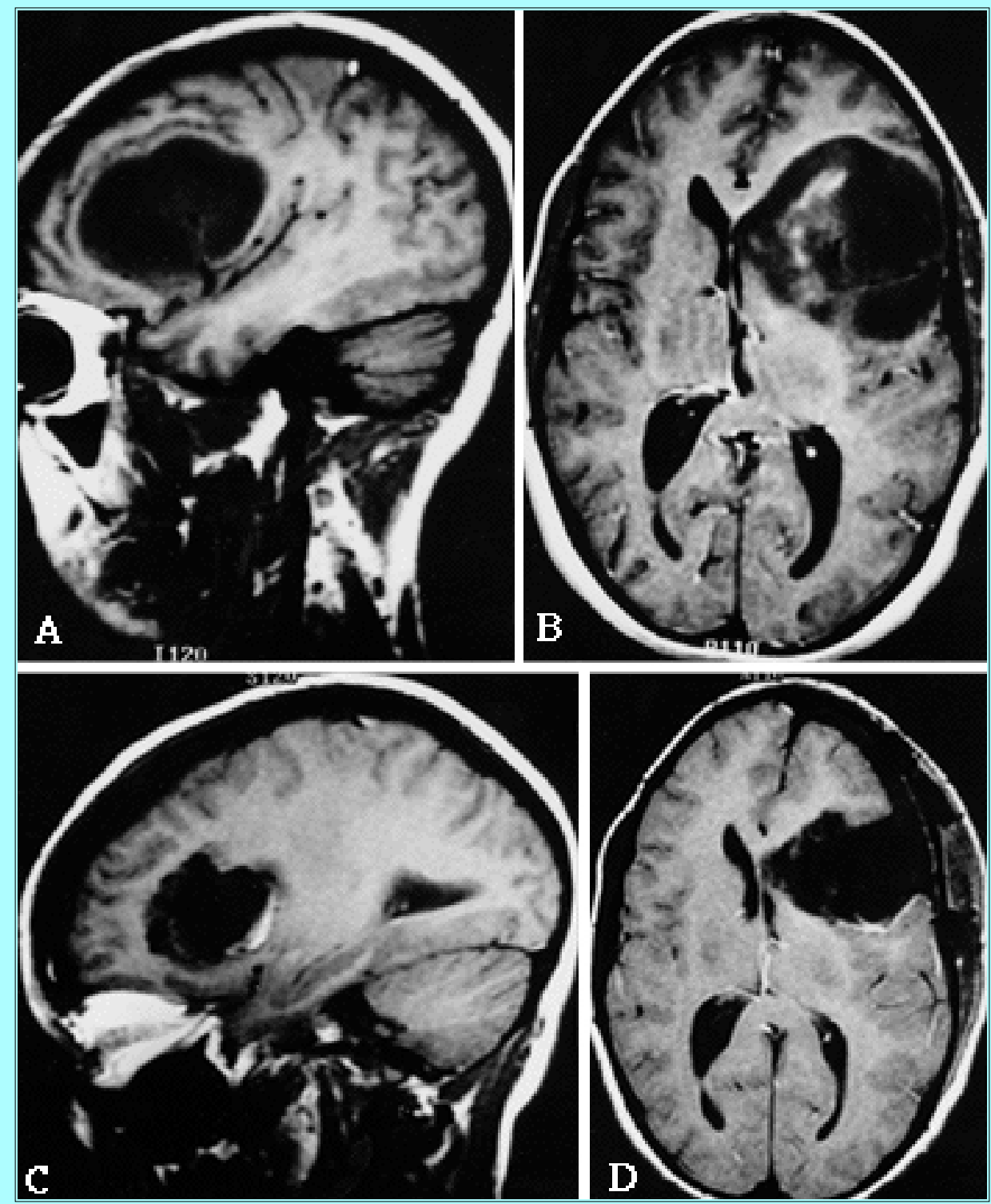

Fig. 3. Three-dimensional reconstruction and cortical mapping showing resection of low-grade astrocytoma impinging on the speech area. A and B: There is a 6 X 6 X $4.5-\mathrm{cm}$ abnormality with heterogeneous signal intensity within the left frontal lobe in this 27 -year-old woman who presented with seizures. It is relatively well defined and has moderate mass effect. There is some feathery enhancement centrally and medially. C and D: Large operative cavity with irregularities along the inner wall that likely represent a small amount of hemorrhage or Surgicel. Enhancement along the posterior aspect of the cavity is suspicious for residual tumor.

Typically, planning neurosurgical procedures is based on the visual interpretation of conventional MR imaging and transformation of this data into 3-D space, which is usually performed mentally by neurosurgeons and neuroradiologists. We have improved the presentation of data for preoperative surgical planning by the following methods: 1) acquiring data with optimum spatial and contrast resolution; 2) identifying relevant anatomical structures, using a combination of automated and manual image processing; and 3) creating high-quality 3-D renderings, which can be easily manipulated at interactive speeds.

Three-dimensional imaging is particularly useful in visualizing tumors that might be related to sensory, motor, visual, or speech areas; those that are deeply located; and in following lesions with radiation 
therapy.[11] The technique offers significant advantages over cross-sectional tomographic images, especially in defining the relationship of tumor to surrounding brain anatomy, including blood vessels, cranial nerves, thalamus, and white matter tracts. The surgeon can measure linear distances and volumes.

We use automated procedures to minimize the amount of interactive manual refinement of the segmentation process. A prototype hardware accelerator allows subsecond rendering of the structures. In this interactive environment, the operator can simulate arbitrary cuts, change the plane, and turn on and off the different structures. For planning surgical procedures, such interactive analysis provides a new level of flexibility greater than that previously reported.[33-35] The ability to generate detailed 3-D renderings at high speed is essential for the interactive exploration and planning sessions; the surgeon can view different approaches and different relationships of the lesions to vessels and cranial nerves and can use the planning process as an important preview of the surgery. Improvements on the system will facilitate the use of 3-D reconstruction to guide intraoperative navigation, allowing one kind of frameless stereotaxy. Elastic deformation of the brain can be simulated interactively to compensate for distortions and displacements during surgery.

The most exciting and important modality, however, is the use of intraoperative MR imaging which is a major advance in the management of low-grade tumors.[8] A collaborative effort between BMH and General Electric Medical Systems resulted in the design of a novel MR surgical suite. The new open-configuration, superconducting 0.5 tesla intraoperative MR imaging system allows direct surgical access to the patient during imaging and provides near real-time, high-quality MR images during the surgical procedure. This assistance to surgical navigation through the 3-D volume of the human brain, with the readily updated images it provides, surpasses the limitation of systems used in the past, in which the images were not updated intraoperatively without moving the patient to a CT or MR imaging suite. When using framed or frameless stereotactic apparatuses during the procedure, cerebrospinal fluid spaces are opened and tissues removed, resulting in shift of this region of the brain and of the pathological tissue; it is obvious that as the procedure progresses the enhanced information provided by the image guidance system becomes less reliable, because it refers to images acquired preoperatively. With intraoperative MR imaging, however, the surgeon follows the progression of the procedure and the extent of tumor resection in a way that allows identification and evaluation of exact localization, percentage of resection, and possible complications.

We performed our first craniotomy for a brain tumor (a temporal ganglioglioma) in August 1996, and since then, the low-grade glioma is the most commonly surgically treated tumor type in the MR suite. We have found this method extremely helpful in designing the surgical approach (craniotomy, point of entry), identifying normal and abnormal anatomy, and in the demarcation of the tumor from the adjacent normal tissue, especially after the administration of contrast material. In this way the procedure is controlled and unexpected complications are identified and rectified, thus leading to reduced morbidity rates.

Based on our experience and on the fact that what we have witnessed in the operating room is just the beginning of the clinical application of a new tool, we believe that the future of intraoperative MR imaging looks bright and will revolutionize the surgical approach, with its excellent tissue discrimination, multiplanar real-time imaging, excellent 3-D reconstruction accuracy, and adequate access to approach any neurosurgically treatable lesion.[53]

\section{References}


1. Bartolomei JC, Christopher S, Vives K, et al: Low-grade gliomas of chronic epilepsy: a distinct clinical and pathological entity. J Neurooncol 34:79-84, 1997

2. Berger MS, Deliganis AV, Dobbins J, et al: The effect of extent of resection on recurrence in patients with low grade cerebral hemisphere gliomas. Cancer 74:1784-1791, 1994

3. Berger MS, Kincaid J, Ojemann GA, et al: Brain mapping techniques to maximize resection, safety, and seizure control in children with brain tumors. Neurosurgery 25:786-792, 1989

4. Berger MS, Ojemann GA: Intraoperative brain mapping techniques in neuro-oncology. Stereotact Funct Neurosurg 58:153-161, 1992

5. Berger MS, Ojemann GA, Lettich E: Neurophysiological monitoring during astrocytoma surgery. Neurosurg Clin North Am 1:65-80, 1990

6. Berger MS, Rostomily RC: Low grade gliomas: functional mapping resection strategies, extent of resection, and outcome. J Neurooncol 34:85-101, 1997

7. Black P, Nikas D: Neurosurgical considerations in low-grade gliomas. 11th International Congress of Neurological Surgery. Amsterdam, The Netherlands, 6-11 July 1997. Bologna: Monduzzi, 1997, pp 371-374

8. Black PM: Surgery for astrocytomas, in Black PM, Schoene WC, Lampson LA (eds): Astrocytomas: Diagnosis, Treatment and Biology. Boston: Blackwell Scientific, 1993, pp 59-72

9. Black PM, Moriarty T, Alexander E III, et al: Development and implementation of intraoperative magnetic resonance imaging and its neurosurgical applications. Neurosurgery 41:831-845, 1997

10. Black PM, Ronner SF: Cortical mapping for defining the limits of tumor resection. Neurosurgery 20:914-919, 1987

11. Buchner H, Adams L, Knepper A, et al: Preoperative localization of the central sulcus by dipole source analysis of early somatosensory evoked potentials and three-dimensional magnetic resonance imaging. J Neurosurg 80:849-856, 1994

12. Bullard DE, Rawlings CD III, Phillips B, et al: Oligodendroglioma. An analysis of the value of radiation therapy. Cancer 60:2179-2188, 1987

13. Burger PC, Vogel SF, Green SB, et al: Glioblastoma multiforme and anaplastic astrocytoma: pathologic criteria and prognostic implications. Cancer 56:1106-1111, 1985

14. Celli P, Nofrone I, Palma L, et al: Cerebral oligodendroglioma: prognostic factors and life history. Neurosurgery 35:1018-1034, 1994

15. Cohadon F, Aouad N, Rougier A, et al: Histologic and non-histologic factors correlated with survival time in supratentorial astrocytic tumors. J Neurooncol 3:105-111, 1985

16. Danks RA, Rogers M, Aglio LS, et al: Patient tolerance of craniotomy performed with the patient under local anesthesia and monitored conscious sedation. Neurosurgery 42:28-34, 1998

17. Daumas-Duport C, Meder JF, Monsaingeon V, et al: Cerebral gliomas: malignancy, limits and spatial 
configuration. Comparative data from serial stereotaxic biopsies and computed tomography (a preliminary study based on 50 cases). J Neuroradiology 10:51-80, 1983

18. Daumas-Duport C, Scheithauer B, O'Fallon J, et al: Grading of astrocytomas. A simple and reproducible method. Cancer 62:2152-2165, 1988

19. Daumas-Duport C, Varlet P, Tucker ML, et al: Oligodendrogliomas. Part I: Patterns of growth, histological diagnosis, clinical and imaging correlations: a study of 153 cases. J Neurooncol 34:37-59, 1997

20. Dropcho EJ, Soong SJ: The prognostic impact of prior low grade histology in patients with anaplastic gliomas: a case-control study. Neurology 47:684-690, 1996

21. Elvidge AR: Long-term survival in the astrocytoma series. J Neurosurg 28:399-404, 1968

22. Fazekas JT: Treatment of grades I and II brain astrocytomas. The role of radiotherapy. Int J Radiat Oncol Biol Phys 2:661-666, 1977

23. Firsching R, Tieben R, Schroder R, et al: Long-term prognosis of low-grade astrocytoma. Zentralbl Neurochir 55:10-15, 1994

24. Gallen CC, Sobel DE, Waltz T, et al: Noninvasive presurgical neuromagnetic mapping of somatosensory cortex. Neurosurgery 33:260-268, 1993

25. Garcia DM, Fulling KH: Juvenile pilocytic astrocytoma of the cerebrum in adults. A distinctive neoplasm with favorable prognosis. J Neurosurg 63:382-386, 1985

26. Garcia DM, Fulling KH, Marks JE: The value of radiation therapy in addition to surgery for astrocytomas of the adult cerebrum. Cancer 55:919-927, 1985

27. Go KG, Keuter EJ, Kamman RL, et al: Contribution of magnetic resonance spectroscopic imaging and L-[1-11C]tyrosine positron emission tomography to localization of cerebral gliomas for biopsy.

Neurosurgery 34:994-1002, 1994

28. Gol A: The relatively benign astrocytomas of the cerebrum. A clinical study of 194 verified cases. J Neurosurg 18:501-506, 1961

29. Gregorie EM, Goldring S: Localization of function in the excision of lesions from the sensorimotor region. J Neurosurg 61:1047-1054, 1984

30. Hakim R, Loeffler JS, Anthony DC, et al: Gangliogliomas in adults. Cancer 79:127-131, 1997

31. Janny P, Cure H, Mohr M, et al: Low grade supratentorial astrocytomas. Management and prognostic factors. Cancer 73:1937-1945, 1994

32. Jubelirer SJ, Rubin M, Shim C: An analysis of 38 cases of low-grade cerebral astrocytoma in adults. West Virginia Med J 89:102-105, 1993

33. Kelly PJ: Stereotactic craniotomy. Neurosurg Clin North Am 1:781-799, 1990

34. Kelly PJ, Kall BA, Goerss S, et al: Computer-assisted stereotaxic laser resection of intra-axial brain neoplasms. J Neurosurg 64:427-439, 1986 
35. Kikinis R, Gleason PL, Moriarty TM, et al: Computer-assisted interactive three-dimensional planning for neurosurgical procedures. Neurosurgery 38:640-651, 1996

36. Kleihues P, Burger PC, Scheithauer BW: Histological Typing of Tumors of the Central Nervous System, ed 2. Berlin: Springer-Verlag, 1993, pp 11-30

37. Kondziolka D, Lunsford LD, Martinez AJ: Unreliability of contemporary neurodiagnostic imaging in evaluating suspected adult supratentorial (low-grade) astrocytoma. J Neurosurg 79:533-536, 1993

38. Lang FF, Epstein FJ, Ransohoff J: Central nervous system gangliogliomas. Part 2: Clinical outcome. J Neurosurg 79:867-873, 1993

39. Laws E Jr: Neurosurgical management of low-grade astrocytoma of the cerebral hemisphere. J Neurosurg 63:819, 1985 (Letter)

40. Laws E Jr: Radical resection for the treatment of glioma. Clin Neurosurg 42:480-487, 1995

41. Laws E Jr, Taylor WF, Bergstralh EJ, et al: The neurosurgical management of low-grade astrocytoma. Clin Neurosurg 33:575-588, 1986

42. Laws E Jr, Taylor WF, Clifton MB, et al: Neurosurgical management of low-grade astrocytoma of the cerebral hemispheres. J Neurosurg 61:665-673, 1984

43. Leibel SA, Sheline GE, Wara WM, et al: The role of radiation therapy in the treatment of astrocytomas. Cancer 35:1551-1557, 1975

44. LeRoux PD, Berger MS, Ojemann GA, et al: Correlation of intraoperative ultrasound tumor volumes and margins with preoperative computerized tomography scans. An intraoperative method to enhance tumor resection. J Neurosurg 71:691-698, 1989

45. Levy LF, Eldvidge AR: Astrocytoma of the brain and spinal cord. A review of 176 cases, 1940-1949. J Neurosurg 13:413-443, 1956

46. Lindegaard KF, Mork SJ, Eide GE, et al: Statistical analysis of clinicopathological features, radiotherapy, and survival in 170 cases of oligodendroglioma. J Neurosurg 67:224-230, 1987

47. Lunsford LD, Somaza S, Kondziolka D, et al: Survival after stereotactic biopsy and irradiation of cerebral nonanaplastic, nonpilocytic astrocytoma. J Neurosurg 82:523-529, 1995

48. Mahaley MS Jr, Mettlin C, Natarajan N, et al: National survey of patterns of care for brain-tumor patients. J Neurosurg 71:826-836, 1989

49. McCormack BM, Miller DC, Budzilovich GN, et al: Treatment and survival of low-grade astrocytoma in adults--1977-1988. Neurosurgery 31:636-642, 1992

50. Medbery CD III, Straus KL, Steinberg SM, et al: Low-grade astrocytomas: treatment results and prognostic variables. Int J Radiat Oncol Biol Phys 15:837-841, 1988

51. Miralbell R, Balart J, Matias-Guiu X, et al: Radiotherapy for supratentorial low-grade gliomas: results and prognostic factors with special focus on tumour volume parameters. Radiother Oncol 27:112-116, 1993 
52. Morantz RA: Radiation therapy in the treatment of cerebral astrocytoma. Neurosurgery 20:975-982, 1987

53. Moriarty TM, Kikinis R, Jolesz FA, et al: Magnetic resonance imaging therapy. Intraoperative MR imaging. Neurosurg Clin North Am 7:323-331, 1996

54. Nakajima S, Atsumi H, Kikinis R, et al: Use of cortical surface vessel registration for image-guided neurosurgery. Neurosurgery 40:1201-1208, 1997

55. Nazzaro JM, Neuwelt EA: The role of surgery in the management of supratentorial intermediate and high-grade astrocytomas in adults. J Neurosurg 73:331-344, 1990

56. Nicolato A, Gerosa MA, Fina P, et al: Prognostic factors in low-grade supratentorial astrocytomas: a uni-multivariate statistical analysis in 76 surgically treated adult patients. Surg Neurol 44:208-223, 1995

57. Nikas DC, Danks RA, Black PM: Tumor surgery under local anesthesia. Tech Neurosurg (In press, 1988)

58. North CA, North RB, Epstein JA, et al: Low-grade cerebral astrocytomas. Survival and quality of life after radiation therapy. Cancer 66:6-14, 1990

59. Ojemann G, Ojemann J, Lettich E, et al: Cortical language localization in left, dominant hemisphere. An electrical stimulation mapping investigation in 117 patients. J Neurosurg 71:316-326, 1989

60. Orrison WW Jr: 3M Mayneord memorial lecture: functional brain imaging--an overview. Br J Radiol 69:493-501, 1996

61. Piepmeier J, Christofer S, Spencer D, et al: Variations in the natural history and survival of patients with supratentorial low-grade astrocytomas. Neurosurgery 38:872-879, 1996

62. Piepmeier JM: Observations on the current treatment of low-grade astrocytic tumors of the cerebral hemispheres. J Neurosurg 67:177-181, 1987

63. Philippon JH, Clemenceau SH, Fauchon FH, et al: Supratentorial low-grade astrocytomas in adults. Neurosurgery 32:554-559, 1993

64. Recht LD, Lew R, Smith TW: Suspected low-grade glioma: is deferring treatment safe? Ann Neurol 31:431-436, 1992

65. Reichenthal E, Feldman Z, Cohen ML, et al: Hemispheric supratentorial low-grade astrocytoma. Neurochirurgia 35:18-22, 1992

66. Rutten EH, Kazen I, Slooff JL, et al: Post operative radiation therapy in the management of brain astrocytoma--retrospective study of 142 patients. Int J Radiat Oncol Biol Phys 7:191-195, 1981

67. Salcman M: The natural history of low-grade gliomas, in Apuzzo MLJ (ed): Benign Cerebral Glioma. Park Ridge, IL: American Association of Neurological Surgeons, 1995, Vol 1, pp 213-229

68. Salcman M: Radical surgery for low-grade glioma. Clin Neurosurg 36:353-366, 1990

69. Sawaya R, Tew JM: Laser Applications in Neurosurgery. Philadelphia: Hanley \& Belfus, 1987 
70. Scanlon PW, Taylor WF: Radiotherapy of intracranial astrocytomas: analysis of 417 cases treated from 1960 thorough 1969. Neurosurgery 5:301-398, 1979

71. Schuurman PR, Troost D, Verbeeten B Jr, et al: 5-year survival and clinical prognostic factors in progressive supratentorial diffuse "low-grade" astrocytoma: a retrospective analysis of 46 cases. Acta Neurochir 139:2-7, 1997

72. Shaw EG, Daumas-Duport C, Scheithauer BW, et al: Radiation therapy in the management of low-grade supratentorial astrocytomas. J Neurosurg 70:853-861, 1989

73. Shaw EG, Scheithauer BW, Gilbertson DT, et al: Postoperative radiotherapy of supratentorial low-grade gliomas. Int J Radiat Oncol Biol Phys 16:663-668, 1989

74. Shaw EG, Scheithauer BW, O'Fallon JR, et al: Mixed oligoastrocytomas: a survival and prognostic factor analysis. Neurosurgery 34:577-582, 1994

75. Shaw EG, Scheithauer BW, O'Fallon JR, et al: Oligodendrogliomas: the Mayo Clinic experience. J Neurosurg 76:428-434, 1992

76. Shibamoto Y, Kitakabu Y, Takahashi M, et al: Supratentorial low-grade astrocytoma. Correlation of computed tomography findings with effect of radiation therapy and prognostic variables. Cancer 72:190-195, 1993

77. Soffietti R, Chio A, Giordana MT, et al: Prognostic factors in well-differentiated cerebral astrocytomas in the adult. Neurosurgery 24:686-692, 1989

78. Steiger HJ, Markwalder RV, Seiler RW, et al: Early prognosis of supratentorial grade 2 astrocytomas in adult patients after resection or stereotactic biopsy. An analysis of 50 cases operated on between 1984 and 1988. Acta Neurochir 106:99-105, 1990

79. Taphoorn MJ, Heimans JJ, Snoek FJ, et al: Assessment of quality of life in patients treated for low-grade glioma: a preliminary report. J Neurol Neurosurg Psychiatry 55:372-376, 1992

80. Taphoorn MJ, Schiphorst AK, Snoek FJ, et al: Cognitive functions and quality of life in patients with low-grade gliomas: the impact of radiotherapy. Ann Neurol 36:48-54, 1994

81. Theunissen PH, Blaauw G: Proliferating cell nuclear antigen immunostaining and survival in cerebral astrocytoma. Histopathology 23:75-79, 1993

82. Touboul E, Schlienger M, Buffat L, et al: Radiation therapy with or without surgery in the management of low-grade brain astrocytomas. A retrospective study of 120 patients. Bull Cancer Radiother 82:388-395, 1995

83. Van Wagenen WPL: Verified brain tumors. End results of one hundred and forty-nine cases eight years after operation. JAMA 102:1454-1458, 1934

84. Vertosick FT Jr, Selker RG, Arena VC: Survival of patients with well-differentiated astrocytomas diagnosed in the era of computed tomography. Neurosurgery 28:496-501, 1991

85. Wallner KE, Gonzales M, Sheline GE: Treatment of oligodendrogliomas with or without postoperative irradiation. J Neurosurg 68:684-688, 1988 
86. Watanabe E, Mayanagi Y, Kosugi Y, et al: Open surgery assisted by the neuronavigator, a stereotactic, articulated, sensitive arm. Neurosurgery 28:792-800, 1991

87. Whitton AC, Bloom HJ: Low grade glioma of the cerebral hemispheres in adults: a retrospective analysis of 88 cases. Int J Radiat Oncol Biol Phys 18:783-786, 1990

Manuscript received March 15, 1998.

Accepted in final form March 23, 1998.

Address reprint requests to: Dimitrios C. Nikas, M.D., Brigham \& Women's Hospital, Children's Hospital, 221 Longwood Avenue/LMRC-121, Boston, Massachusetts 02115. email: dnikas@ rics.bwh.harvard.edu. 